\title{
A Modular User Interface of Robots
}

\author{
Ji hwan Park, Tae Houn Song, Key Ho Kwon, and Jae Wook Jeon
}

\author{
School of Information and Communication Engineering, Sungkyunkwan University \\ Chunchun-dong 300, Jangan-gu, Suwon 440-746, South Korea \\ fellens@gmail.com, thsong@ece.skku.ac.kr, \\ \{khkwon, jwjeon\} @yurim.skku.ac.kr
}

\begin{abstract}
This paper discusses development of a control system for home robots. When a user requests a user interface for home robot, the home robot sends this robot's user interface to the user's PDA. Then, the robot can be efficiently controlled from this user interface on the PDA. The home server distributes the service of robots, and manages the transmission of service interface modules. When this procedure is used, home network traffic is reduced, and the user can simply access a service of a robot.
\end{abstract}

Keywords: user interface, robot, UI, interface module, robot interface.

\section{Introduction}

As robots evolve, robots are increasing becoming move widely used for a variety of functions. This increased usage makes robots' interface programs very important. A robot generally is in need of special controllers or application programs. When a robot is controlled by using a specific controller, the mobility and expandability of the robot can be attenuated. When a user installs an application program for robot control from a PC, the resource of the PC is wasted. In many situations of robot control, the user must re-install the application program when the PC changes. Also, A PC is not mobile whereas a PDA is. The user interface programs used for robots are not necessarily similar for each robot. So a user must study several new user interfaces when robots change. In previous work, the robot control method via internet is showed good performance [1-2].

Most robots operate and think independently of other robots. However, robot technology and computer networks are developing rapidly, and many research organizations have attempted to integrate robot technology and communication networks. This research assumes that robots interact with each other or a computer network, and can perform a large number of functions [3].

In this paper, we propose a PDA based user interface module management system for a home service robot. The user interface is divided into service modules and standard interface modules. These modules are made from XML. The robot language to control and manage robots is many. XML based RobotML exist of them [4]. RobotML is suitability and useful language at internet environment. And XML is simple grammar and very easy. Also data can be expressed intuition [5]. 
A home server manages the service robot via a home network. The home server has a service list for the service robot which is connected to the home server through a network. When a user connects to the home server by means of the user's PDA and then requests any service, the home server searches for the robot which has the requested service. The home server then transfers this robot's user interface module and service list to the user's PDA. We assume that the robot has a powerful CPU, large data storage, and a service interface module for this proposal. Also we assume that the command protocol is standardized.

Then, when we use this system and connect to the network, we can control any robot anywhere on the network. When a user requests a service, the home server transfers the service interface module of the robot which has the requested service. So a user can control the robot anywhere he or she has access to the network. Also resources of the home server are saved because it is necessary that a user interface of service robots be installed.

Section 2 describes the interface transmission system. Section 3 describes the service interface module and standard interface module. Section 4 describes a result of the user interface program; Section 5 provides a conclusion and presents some directions for future work.

\section{Interface Transmission System}

We now describe the user interface modules management system for a robot. The robot interface program consists of many different interface modules. A security robot consists of a remote supervising computer, a security module, application modules, a RF interface, and GSM controller modules. These components of the robot system manage the robot function [6]. In our work described in this paper, the modules are grouped into standard interface modules and service interface modules and then managed. The standard interface modules have the common function of the service robot and are stored in a user's PDA.

In this paper, we assume that the command protocol is standardized. So the standard interface module can control all robots. The service interface module controls special functions. The service interface modules are stored in the service robots or on the home server. When the service interface module is requested, the service interface modules are transferred to the user's PDA.

\subsection{Home Server and Service Robots}

Classically, a home server controls the electronic products in a home that are connected to the home server [7, 8]. In our work, the home server controls home service robots also. A user's PDA is connected to the home server through a home network. So, the role of home server is that of a bridge between the user and the service robots $[7,8]$. When the service robot requests registration, the home server gives an ID to the robot and requests a service list from the service robot. When the home server receives the service list, the home server registers and renews the service list of the home server. Then the home server has a service list for all of the robots. 
When a user selects any service, the home server searches the service list of the home server for the requested service. The home server then links the user with the robot which has the requested service and higher priority.

A service robot supports services for a user. The service robots have a service list which is list of the supported services for the robots. The service list is transferred from the service robot to the home server. The service robot has a service interface module which is adequate to support the service list. This module is implemented using XML and has command protocols and a layout of a user interface.

\subsection{Personal Digital Assistant (PDA)}

The user must select the controller which controls the service robot. A PDA is widely used and has more mobility than other possible control devices. A PDA can connect to a network to using a wireless LAN. Because of this possibility, a PDA can be a good controller. When a user connects to a home server, the home server transfers a service list from the home server to the user's PDA. A user can select services that are indicated in a black color, and not select services that are listed in a gray color. The gray color indicating unavailability of a service means that another is using that service.

When a user selects a service, the user's PDA requests the selected service. After, the home server searches for a robot which supports this requested service. The home server then transfers a service list and service interface module for the robot to the requesting user's PDA. Then the user's PDA that received these components builds a user interface program with the service list, the service interface module, and the standard interface module.

\subsection{Interface Transmission}

Figure 1 shows a flow chart of the transfer of the service interface module to the user's PDA. First, when a service robot is powered on, the service robot checks the home network periodically. When a home server is found, the service robot connects to the network and requests registration on the home server. To control the robot, a user must connect to the home server. The service robot is also connected to the home server and has one service or more. When the service robot requests a service registration on the home server, the home server gives an ID to the robot. Then, the home server demands a service list of the service robot. The service list has all the possible functions for the service robot. When a service robot transfers its service list to the home server, the home server registers and renews the service list of the home server. The home server decides priorities of all the robots.

To control any service robot, a user must connect to the home server. When a user's PDA is connected to a home server, the home server can transfer a requested service list. Then a user will select which service to use. The PDA requests service to the home server. The home server then requests a service search for a robot which is supported and has a highest priority. If the service robot is found, the home server requests this service robot's service list and the service interface module of this service robot. Then, the service robot transfers a service list and a service interface module to the home server. The home server which received the service list and 


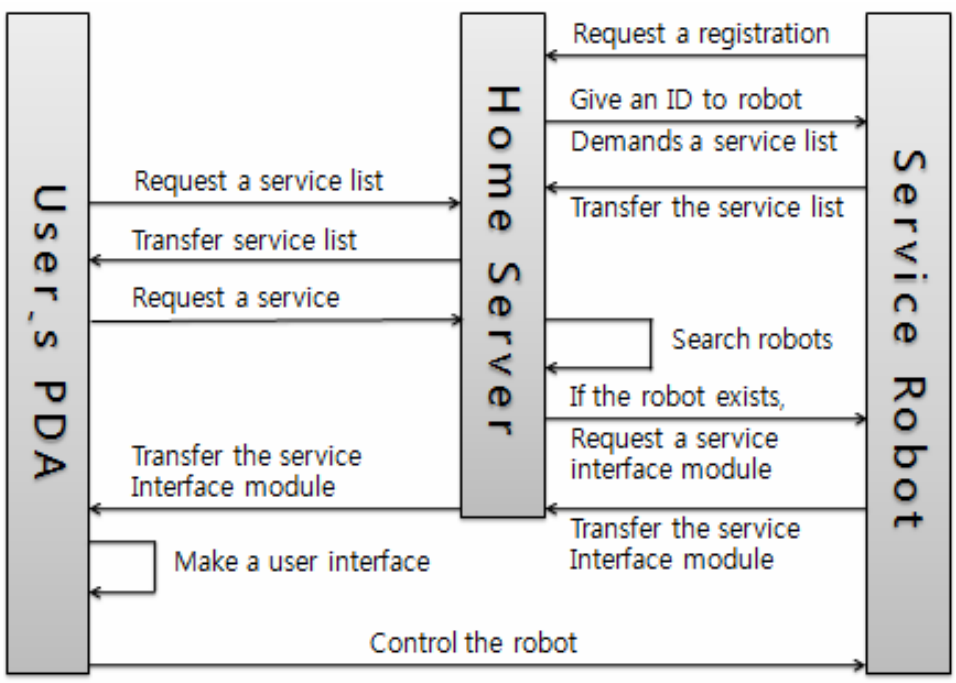

Fig. 1. A flow chart for the interface transmission system

service interface module then transfers these components of the service robot to the requesting user's PDA. This user's PDA, upon receiving these components, builds a user interface program into these components and standard interface module.

Even if a user requests the same service at another time, a new user interface is built as it is may not the same as a prior time, because the robot which is now selected may be different.

\section{Interface Modules}

In our work described in this paper, a user interface is in the user's PDA. This user interface is divided into service modules and standard interface modules. The standard interface module is a common part of the interface. The service interface module is a module transferred to a service robot. Table 1 show that many robots may have common services. A large number of robots have movement, rotation, voice output, camera output, and a range sensor service [9-18].

Table 1. The common function of robots

\begin{tabular}{l|cccc}
\hline & Movement & Voice Output & Camera Output & Range sensor \\
\hline Patrol robot(5) & 5 & 3 & 5 & 5 \\
Cleaning robot(6) & 6 & 1 & 1 & 3 \\
Housework robot(5) & 5 & 4 & 5 & 5 \\
Toy robot(14) & 12 & 3 & 3 & 6 \\
Military robot(10) & 10 & 3 & 8 & 7 \\
\hline Total(40) & 38 & 14 & 22 & 26 \\
\hline
\end{tabular}


According to our research, movement, rotation, voice output, camera output, and range sensor service are constituted as parts of the standard interface module. Other services are constituted in the service interface module. A robot has a service list. The service interface module and the standard interface module are managed using the service list.

\subsection{Service List}

A service list is stored on a service robot and a home server. A service list of the robot is stored only if it is a service of that robot. But a service list of the home server represents service lists of all connected robots. Figure 3 is the architecture of the home server's service list. The service list of the home server is the stored service robots' lists and the priority list of robots. If another user controls any robot, the robot is marked as being down. Then other users cannot control a service robot overlap.

Example of the service list which was produced with XML

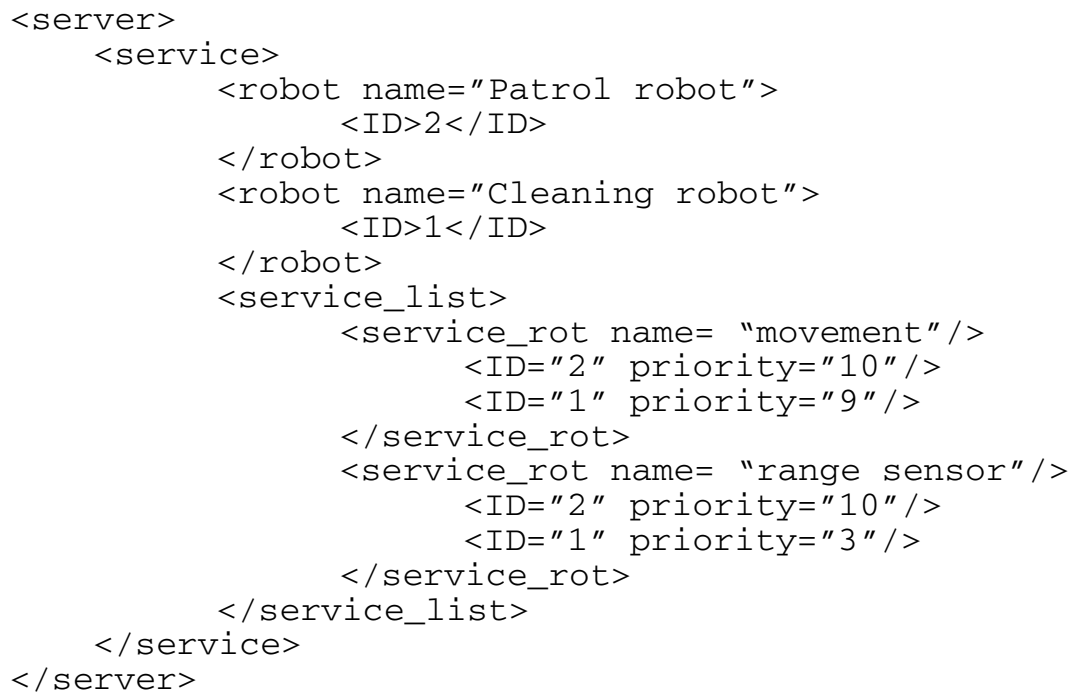

An example is the service list that constitutes XML. The service list of the service robot is different from the service list of the home server. The service list of the home server is stored as a command protocol of all functions of the robot and the supported service. For example, we have camera output, navigation, and patrol service.

\subsection{Interface Modules}

Many robots have common functions. Generally, a robot has functions of movement and rotation. Recently robots support both a voice output and camera output. We define a standard interface module if that module is made with common functions. 
When any robot uses standard protocol which is suitable for a standard interface module, a user can control the robot by using the standard interface module. The standard interface module is contained in the user's PDA.

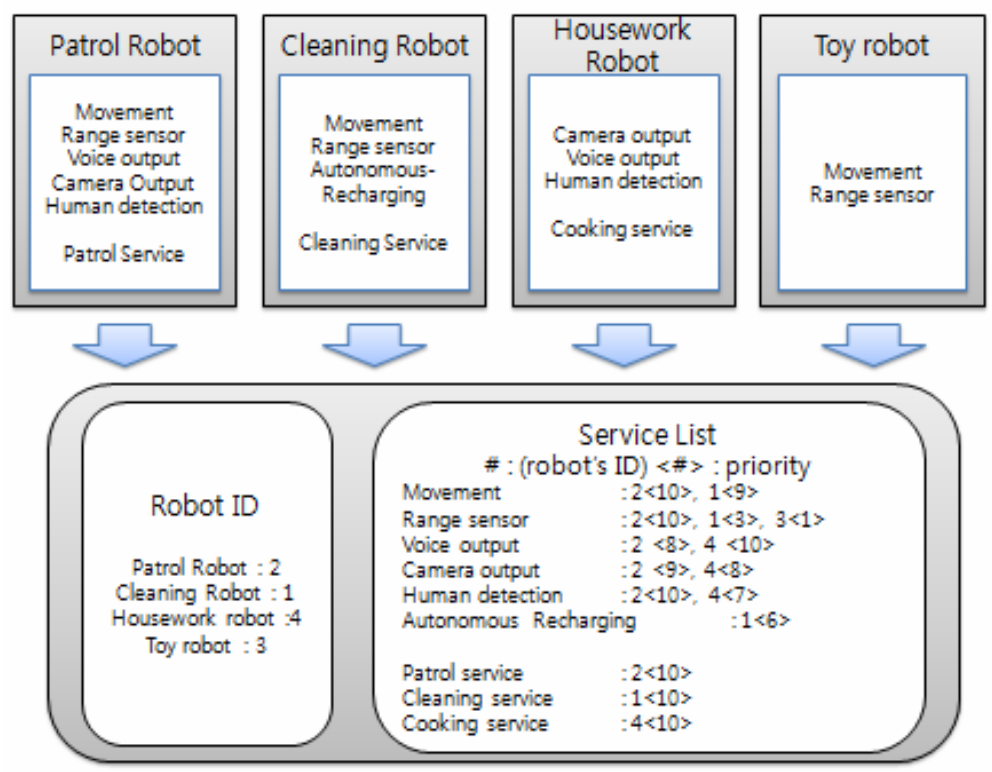

Fig. 2. The architecture of the Service List in a Home server

A service interface module is a special interface module. It supports functions without a standard interface module. Each robot has a different service interface module. The service interface module is implemented using XML. When a home server requests a service interface module, the service interface module is transferred from the robot to the home server. It can use special functions which are not included in the standard interface module. It defines a layout for the user interface. For example, some examples of special functions are the emotion of toy robots or a mine detector of military robots.

\subsection{XML Based User Interface Programs}

The service interface module and the service list are implemented in XML. The service interface module is stored protocols and a layout of a user interface. When the service interface module and the service list is transferred to a user's PDA, a PDA builds a user interface program with the service interface module and standard interface module. When a robot is changed, a robot interface program will be changed. But these are similar interface programs. So, it is easy to learn how to use the slightly changed program. 
Example of a Service Interface Module
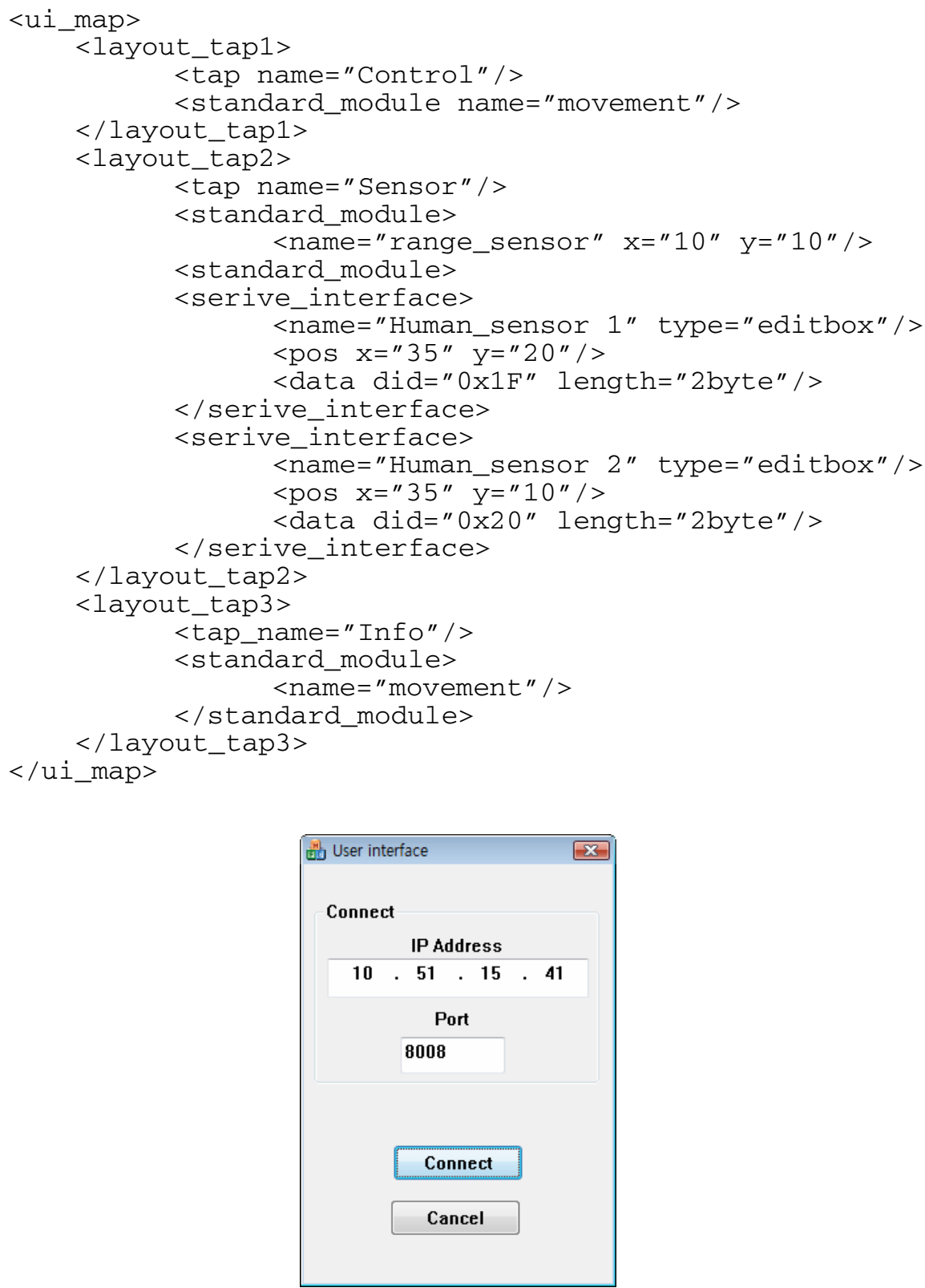

Fig. 3. The home server connection screen of the user interface 


\section{Results}

Figure 4 is a screen showing attempts to connect to a home server. When a user enters the IP of the home server in the edit window, the user interface program tries to connect to the home server. When a user connects to the home server, a user interface program requests the home server to transfer a service list from the home server.

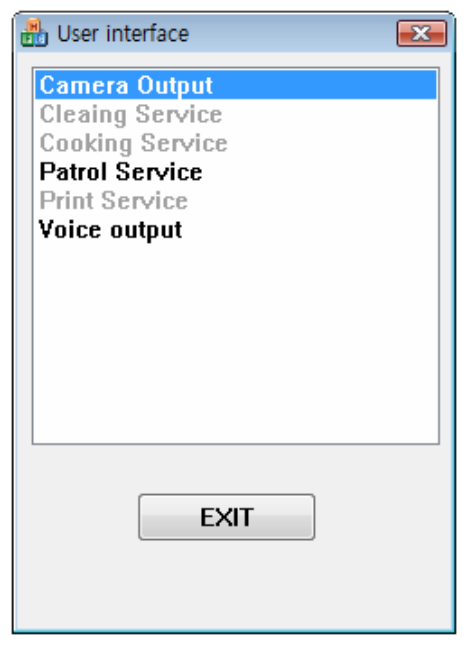

Fig. 4. The service list menu
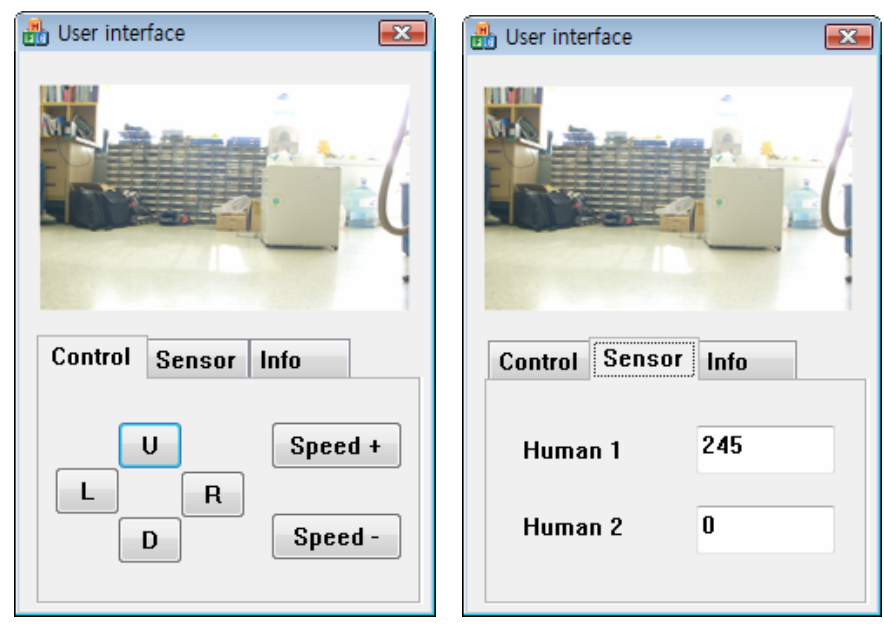

Fig. 5. The user interface program of the robot which is supported the patrol service

When the home server transfers the service list to the user's PDA, the user interface program in the PDA shows a service list screen. Figure 5 show that list screen. Currently, a user only selects the patrol service. The other services - cooking, 
print and the cleaning services - are used by other user. So these services are shown in a gray font.

When a user selects the patrol service, the user interface program in a PDA requests a service interface module and service list of the service robot. Figure 6 is a user interface program which builds a user interface program with these and the standard interface module.

\section{Conclusion}

This paper proposed the configuration of a home server, home network, and interface transmission system for efficient management of the home robot by the home network. When a home server is found, the service robot connects to the network and requests registration on the home server. When the service robot requests a service registration on the home server, the home server gives an ID to the robot. Then, the home server demands a service list of the service robot. The service list has all the possible functions for the service robot. When a service robot transfers its service list to the home server, the home server registers and renews the service list of the home server. The home server decides priorities of all the robots.

A user must connect to the home server. When a user's PDA is connected to a home server, the home server can transfer a requested service list. Then a user will select which service to use. The PDA requests service to the home server. The home server then requests a service search for a robot which is supported and has a highest priority. If the service robot is found, the home server requests this service robot's service list and the service interface module of this service robot. Then, the service robot transfers a service list and a service interface module to the home server. The home server which received the service list and service interface module then transfers these components of the service robot to the requesting user's PDA. This user's PDA, upon receiving these components, builds a user interface program into these components and standard interface module.

The role of the home server is to link robots and user. When new robot is added to the home network, the home server does not need to install the special interface program of the robot. The home server distributes the service of the robots, and manages the transmission of service interface modules. When this procedure is used, home network traffic is reduced, and the user can simply access the service of a robot.

Acknowledgments. This research was supported by the MIC, Korea under ITRC, IITA-2006-(C1090-0603-0046).

\section{References}

1. Suzuki, T., Fujii, T., Yokota, K., Asama, H., Kaetsu, H., Endo, I.: Teleoperation of Multiple Robots through the Internet. Proc. of IEEE Int. Workshop on Robot and Human Communication, pp. 84-89 (1996)

2. Taylor, K., Dalton, B., Trevelyan, J.: Web-Based Telerobotics. Robotica 17, 49-57 (1999) 
3. Jung, B.-c., Park, J.-h., Choi, D.-s., Kim, H.-m.: A study on intelligent robot based on home network. The. Korea Society of Mechanical Engineers autumn collected papers, pp. 792-798 (2003)

4. Makatchev, M., Tso, S.K.: Human-robot interface using agents communicating in an XML-based markup language. Robot and Human Interactive Communication, 2000. ROMAN 2000. Proceedings. 9th IEEE International Workshop, September 27-29, 2000, pp. 270-275 (2000)

5. Young, M.J.: Step by Step XML. Microsoft.

6. Luo, R.C., Hsu, T.Y., Lin, T.Y., Su, K.L.: The development of intelligent home security robot. ICM '05. IEEE International Conference on Publication, July 10-12, 2005, pp. 422 427 (2005)

7. Nakajima, T., Satoh, I.: Personal home server: enabling personalized and seamless ubiquitous computing environments. Pervasive Computing and Communications, pp. 341 345 (2004)

8. Bae, C., Yoo, J., Kang, K., Choe, Y., Lee, J.: Home server for home digital service environments Consumer Electronics. In: ICCE. 2003 IEEE International Conference, June 17-19, 2003, pp. 382-383 (2003)

9. http://www.cybermotion.com/datasheets/sr3esp.pdf

10. http://www.joymecha.com

11. http://imoyo.com/

12. http://www.roombavac.com

13. http://www.roboblock.co.kr

14. http://www.aibotown.co.kr

15. http://www.airobot.com

16. http://irobot.com

17. http://packbot.com

18. http://www.honda.co.jp/ASIMO/ 